\section{ON THE SIMULATION OF} THE FIRST NATURAL FREQUENCY OF A TUNED MASS DISC DAMPER

CHRISTIAN BRECHER, GUIFENG ZHAO, MARCEL FEY

RWTH Aachen University, Laboratory for Machine Tools and Production Engineering, Aachen, Germany

DOI: 10.17973/MMSJ.2021_10_2021091

G.Zhao@wzl.rwth-aachen.de

The tuned mass disc damper (TMDD) can be applied to avoid chatter of machine tools. The effectiveness of the TMDD depends mostly on the tuning accuracy of the natural frequency. In this paper, three finite element models are presented to simulate the vibrations of the TMDDs. The simulated natural frequencies are compared with measured results. The third model solves the problems of the first and the second model and can predict the natural frequencies of the TMDDs with an acceptable error.

KEYWORDS

finite element method, simulation, tuned mass disc damper, natural frequency, impact hammer test

\section{INTRODUCTION}

Chatter is a major problem in machining with machine tools [Taylor 1906]. It limits the productivity and harms the product quality [Munoa 2016]. Many engineers and researchers are committed to find different chatter suppression methods, such as optimization of the machine tool structure, process parameter tuning, spindle speed variation, active damping techniques and passive damping techniques. The passive damping techniques are widely used because they are costeffective and robust, the tuned mass damper (TMD) is one of them [Brecher 2017] [Munoa 2016]. Frahm has proposed the original idea of the TMD [Frahm 1911]. The TMD was first applied as a chatter suppression method by Hahn [Hahn 1951]. One natural frequency of the TMD must be tuned accurately to match the critical natural frequency of the machining system. There are two major drawbacks of the TMD. At first, the effectiveness of the TMD is influenced strongly by the accuracy of the frequency tuning. Furthermore, the damping effect of the TMD is mostly realized by using the viscous materials, which are very sensitive to the external temperature and degrade over time [Li 2017] [Friend 2000]. Alternatively, the particle damper (PD) dissipates vibration energy by the collision and the friction between the particles and the hosting enclosure, hence it is independent of the environmental temperature and very durable [Friend 2000] [Meyer 2020]. However the PD generates lower damping effect than the TMD at the same vibration level [Li 2017]. In order to avoid the drawbacks of the TMD and the PD, a few researchers have explored the tuned mass particle damper (TMPD), which combines the merits of the TMD and the PD [Li 2017] [Liu 2020] [Lu 2020].

The tuned mass disc damper (TMDD) was proposed in the paper [Brecher 2020], which also combines the principles of the TMD and the PD. Fig. 1 shows the design of the TMDD. It is attached to the primary structure through the magnetic foot with a M6 threaded hole, a M6 screw connects to the magnetic foot and works as a cantilever beam. The identical metal discs are installed on the M6 screw, the axial positions of the metal discs along the M6 screw are adjusted by changing the number of the front and back washers. The washers are not pulled together by force, thus the clearances between the screw, the washers and the metal discs are maintained after assembling the TMDD. These clearances are important for the damping effect of the TMDD, which is generated by the collision and the friction between the metal discs and the M6 screw, and it will be strongly amplified in case of an accurate frequency tuning. The natural frequency of the TMDD depends on the total mass and the axial positions of the metal discs. [Brecher 2020]

As shown in Fig. 1, the total mass of the metal discs of the TMDDs used in this paper is $600[\mathrm{~g}]$, the thickness of one washer is 1.5 [mm], and the M6x170 screws are used for the TMDDs. The screw mainly contributes to the stiffness of the TMDD, whereas the metal discs and the washers decides the vibration mass and its position.

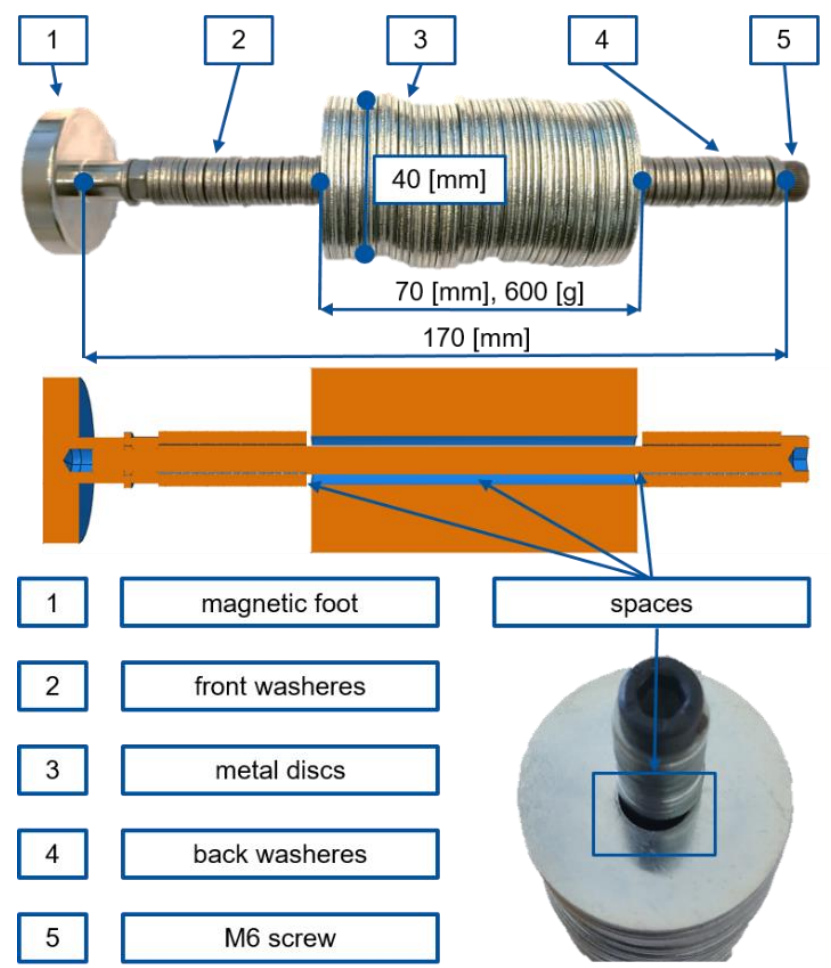

Figure 1. Structure of the TMDD

The frequency response functions (FRF) of the TMDDs are measured and the natural frequencies are extracted from the FRFs [Brecher 2020]. The authors haven't considered the vibration amplitudes of the TMDDs at their natural frequencies due to two facts. First of all, the amplitudes depend on the damping effects of the TMDDs. The damping effects are strongly influenced by the montage process. This means the vibration amplitude of a TMDD is not reproducible after different montage processes. Additionally, an accurate frequency tuning is much more important than the damping effect of a single TMDD. Because of the importance of the frequency tuning for the TMDD, it will be very meaningful to develop a simulation model for predicting the natural frequency. The required positions of the metal discs for the different target frequencies can be predicted by utilizing a plausible simulation model, which can be parameterized with only few measurements. It can save the development time.

This paper is organized as follows: Following this introduction section, section 2 shows the measurements of the TMDDs. Subsequently, three finite element models are presented to predict the natural frequencies of the TMDDs in section 3 . The simulated natural frequencies are compared with measured 
results. Conclusions and outlooks are provided in the last section.

\section{MEASUREMENT}

53 TMDDs are measured in this paper. The TMDDs are identical except the number of the front washers and the number of the back washers. The number of the front washers increases from 1 to 53 , while the number of the back washers decreases from 53 to 1 . The measurement setup is shown in Fig. 2 . The natural frequencies of the TMDDs are measured with the impact hammer tests. An impact hammer excites the structure at the red marked impact point in y direction, and the TMDD vibrates mainly in $y$ direction. Because the washers are not pulled together by force, the washers and discs can move freely in the YZ plane during the measurements. The acceleration signals of the $y$ direction are measured at the free end of the TMDDs. The mass of the acceleration sensor is $9[\mathrm{~g}]$ and is only $1.5 \%$ of the total mass of the metal discs, hence its influence on the measurement is neglected, and it is not considered in the simulation models in the next section.

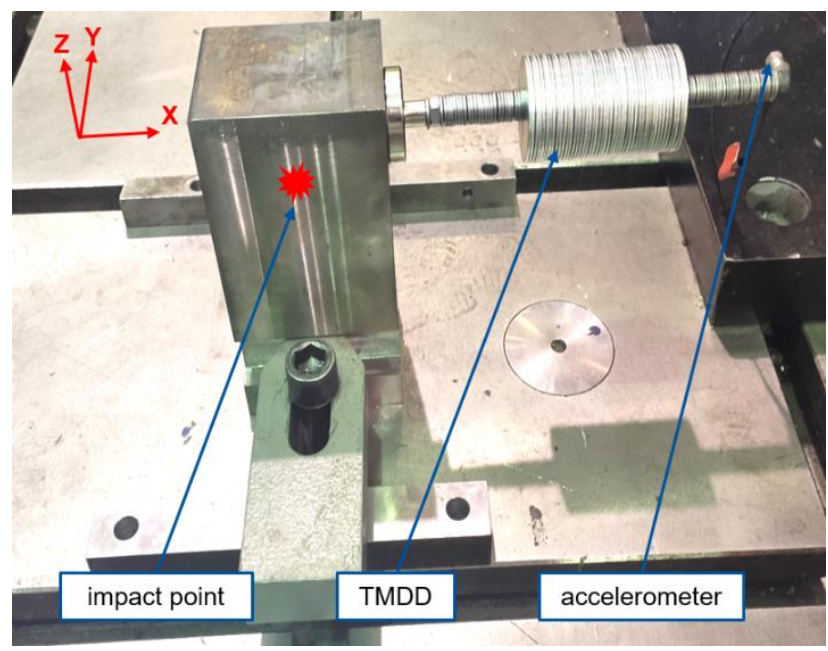

Figure 2. Measurement setup

The measurement results are shown in Fig. 3. Generally, the natural frequency of the TMDD decreases with the increase of the total thickness of the front washers. Three simulation models of the TMDDs will be presented in the next section.

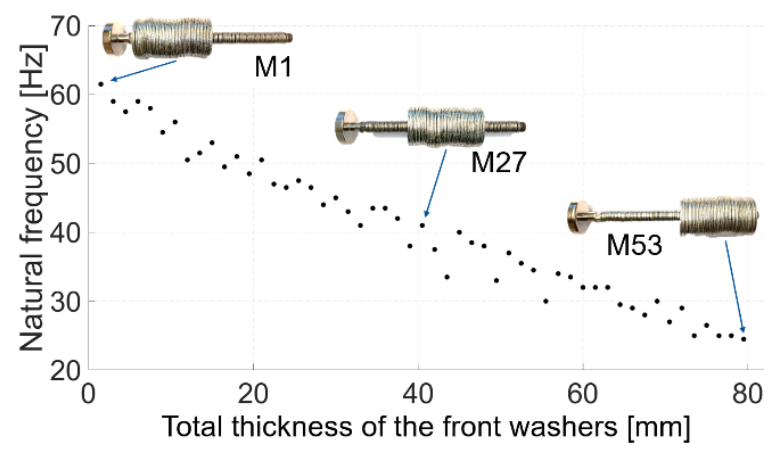

Figure 3. Results of the impact hammer tests

\section{FINITE ELEMENT MODELS OF THE TMDD}

The TMDD has many washers and metal discs, thus it is very time consuming to simulate all of them in detail. The washers shown in Fig. 1 only prevent the motions of the metal discs along the screw and they have negligible influence on the vibration of the
TMDD. On account of that they will not be included in the simulation models. The metal discs are the vibration mass of the TMDD, the abstraction of them has a dominant influence on the simulation results.

The aim of this section is to find a simulation model, which can predict the natural frequencies of the TMDDs. The average difference between the simulated and the measured natural frequencies should be smaller than $3[\mathrm{~Hz}]$. This tolerance range is defined based on two arguments. At first, the natural frequency of one TMDD can vary in an interval with a bandwidth about $3[\mathrm{~Hz}]$ because of the influence of the montage. On account of that a tighter tolerance range is not necessary. Additionally, multiple TMDDs are used simultaneously in most cases, thus the frequency tuning error of the single TMDD can be compensated by each other. In this section, three simulation models are presented. All the models are created with the software abaqus. The screw is considered as a beam with the diameter $6[\mathrm{~mm}]$, and the connection between the screw and the magnet is considered as rigid. The best prediction results can be provided by the third model, which solves the problems of the first and the second models.

\subsection{Metal discs considered as distributed mass along the screw}

In this subsection, the metal discs are considered as local distributed mass along the corresponding part of the screw. The Young's modulus $210[\mathrm{GPa}]$ and Poisson's ratio 0.3 are assigned to the entire model. As shown in Fig. 4, the model is divided into two regions marked with two different colors. Two different densities are applied for them. The standard density $7.9\left[\mathrm{~g} / \mathrm{cm}^{3}\right]$ of the steel is assigned to the blue marked region. Because the metal discs are attached to the red marked region of the screw, an equivalent density $333\left[\mathrm{~g} / \mathrm{cm}^{3}\right]$ is applied for this region, so that the simulation model and the real TMDD have identical mass.

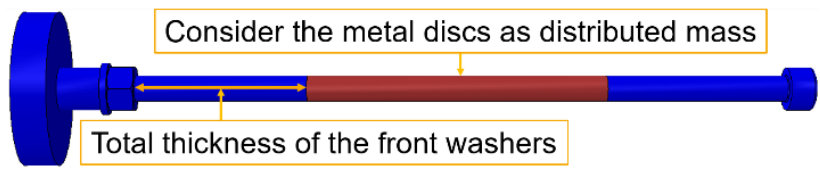

Figure 4. Metal discs considered as distributed mass

The 53 TMDDs mentioned in the second section are simulated. As shown in Fig. 5, the first vibration mode of the model is the first bending mode of the screw, its natural frequency corresponds to the measured natural frequency using the impact hammer method. The simulation results are shown in Fig. 6, the simulated natural frequencies are higher than the measured results. As shown in Fig. 7, the maximum difference between the simulations and the measurements $32[\mathrm{~Hz}]$ occurs with the total thickness of the front washers $3[\mathrm{~mm}]$ and the average difference is $12.5[\mathrm{~Hz}]$. Generally, the difference decreases with the increase of the total thickness of the front washers.

The average difference $12.5[\mathrm{~Hz}]$ is not in the tolerance range $3[\mathrm{~Hz}]$, therefore the accuracy of this simulation model is not enough. The center of mass of the simulation model lies on the axis of the M6 screw, whereas the center of mass of the real TMDD is not on this axis due to the space between the metal discs and the screw. It can be the reason for the differences between the simulations and the measurements. Based on this assumption, another model will be explored in the next subsection. 


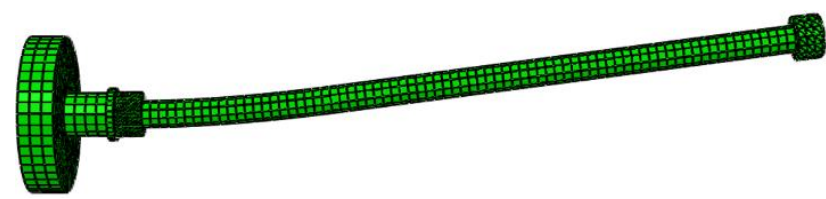

Figure 5. First vibration mode

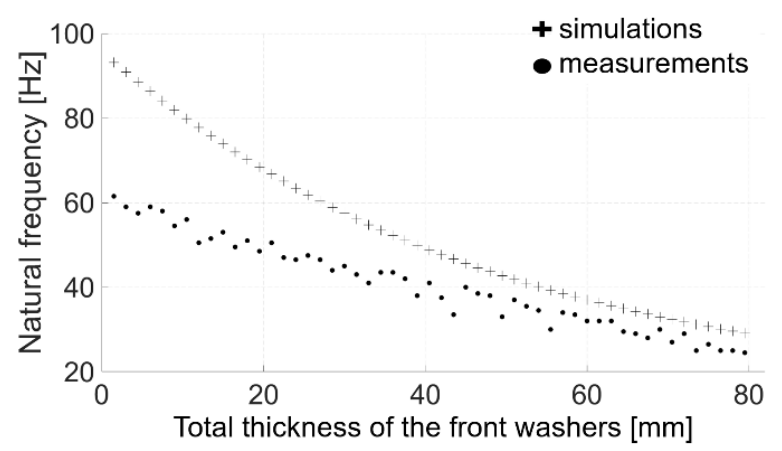

Figure 6. Simulation results considering the metal discs as distributed mass

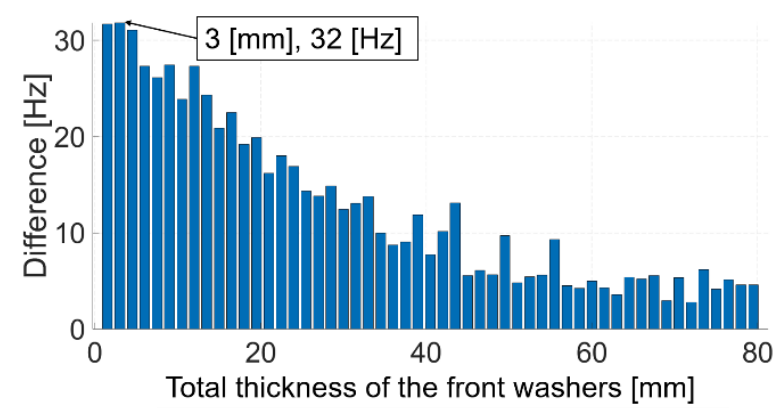

Figure 7. Differences between the simulations and the measurements

\subsection{Metal discs considered as a single cylinder consisting of an isotropic material}

In this subsection, the metal discs are abstracted as a single cylinder, which consists of an isotropic material. As show in Fig. 8, this model takes into account the space between the metal discs and the screw. The model is divided into two regions. The interface between these two regions is simulated as a surface to surface contact. The Young's modulus 210 [GPa] is assigned to the blue marked region, the density $7.9[\mathrm{~g} / \mathrm{cm} 3]$ and the Poisson's ratio 0.3 are assigned to the whole model. The challenge is how to choose the Young's modulus for the red marked cylinder. At first, the standard Young's modulus 210 [GPa] of the steel is used for the cylinder.

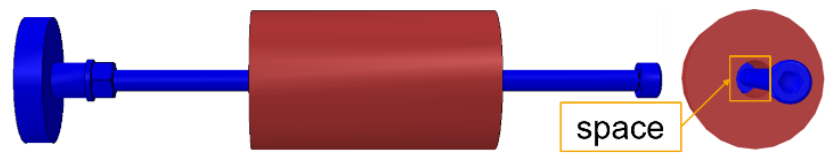

Figure 8. Metal discs considered as a single cylinder

Same as in the last subsection, 53 TMDDs are simulated. The first vibration mode of the model is shown in Fig. 9, it is the first bending mode of the screw. The natural frequency of this mode corresponds to the measured natural frequency in section 2 . As shown in Fig. 10 and 11, the maximum difference between the simulations and the measurements $60.5[\mathrm{~Hz}]$ occurs with the total thickness of the front washers $1.5[\mathrm{~mm}]$, the average difference is $12.8[\mathrm{~Hz}]$. When the total thickness of the front washers exceeds $50[\mathrm{~mm}]$, the model can predict the natural frequencies of the TMMDs with an average difference smaller than $3[\mathrm{~Hz}]$. Otherwise, the simulated natural frequencies are much higher than the measured results. An assumption can explain this phenomenon. The metal discs can actually have relative motions between each other, in contrast the relative motions in the model are restricted by abstracting the metal discs as a single cylinder with the Young's modulus 210 [GPa]. This restriction has increased the stiffness of the model, thus the simulated natural frequencies are higher than the measured results.

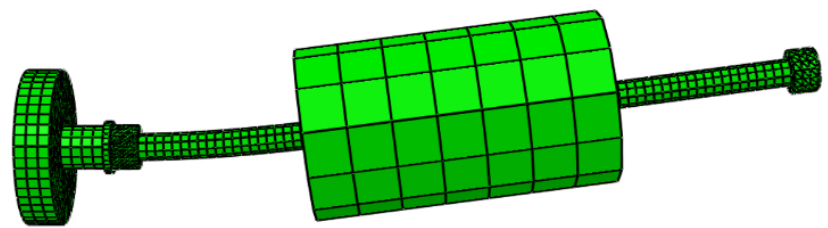

Figure 9. First vibration mode

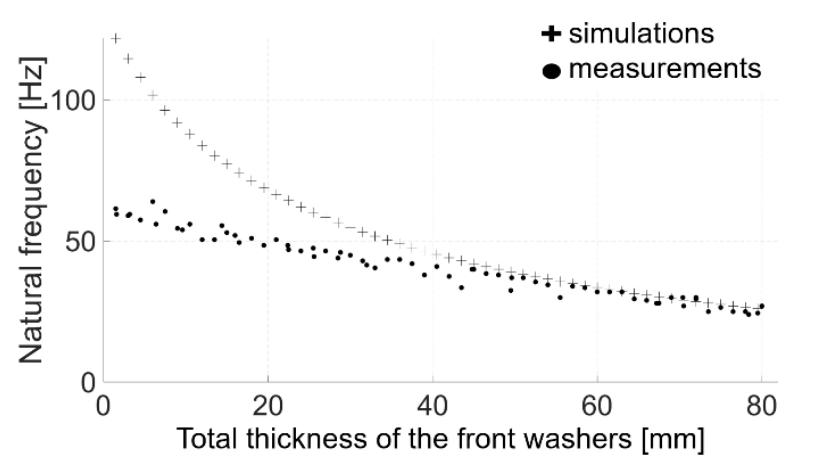

Figure 10. Metal discs considered as a single cylinder with an isotropic material (Young's modulus 210 [MPa])

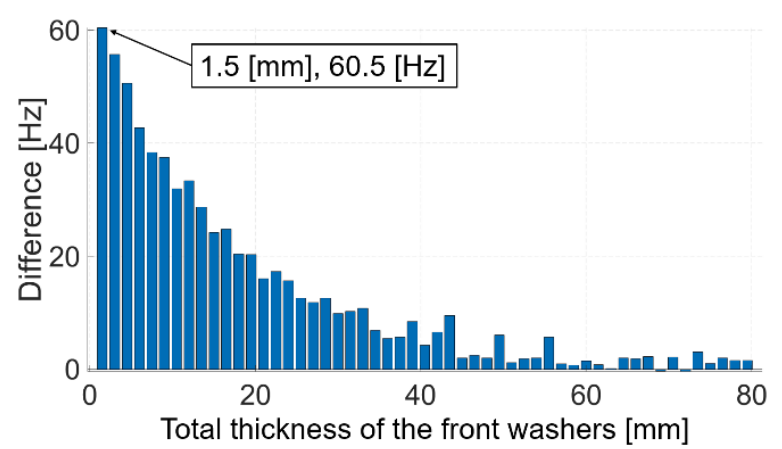

Figure 11. Differences between the simulations and the measurements

Based on the aforementioned assumption, the Young's modulus should be as small as possible. However there is a limit, if the Young's modulus of the cylinder is too small, the first vibration mode of the model is not the first bending mode of the screw, rather the vibration mode of the cylinder as shown in Fig. 12. At this mode, the screw has negligible deformation, whereas the elements of the cylinder deform dramatically due to the very small Young's modulus. It results a problem for the mapping between the simulations and the measurements.

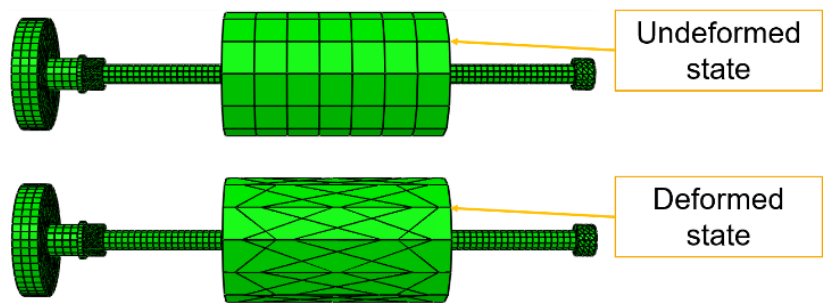

Figure 12. First vibration mode

After several try and error processes, the minimum value of the Young's modulus turns out to be 11 [MPa]. As shown in Fig. 13 
and 14 , the maximum difference between the simulations and the measurements $10[\mathrm{~Hz}]$ occurs with the total thickness of the front washers $12[\mathrm{~mm}]$, the average difference is $3.2[\mathrm{~Hz}]$. The differences between the simulations and the measurements become smaller with the Young's modulus 11 [MPa] than with 210 [GPa]. Additionally, this average difference $3.2[\mathrm{~Hz}]$ is much smaller than the average difference $12.5[\mathrm{~Hz}]$ in the last subsection. However it is still not in the tolerance range $3[\mathrm{~Hz}]$. Two assumptions have been proved in this subsection. At first, the model in this subsection is more accurate than the model in the last subsection, because the space between the metal discs and the screw has been simulated correctly. Furthermore, the model in this subsection with a smaller Young's modulus can provide better simulation results. However the Young's modulus can not be smaller than 11 [MPa] for this model. The possible solution for this problem is using an orthotropic material definition for the cylinder. The model with the orthotropic material definition will be discussed in the next subsection.

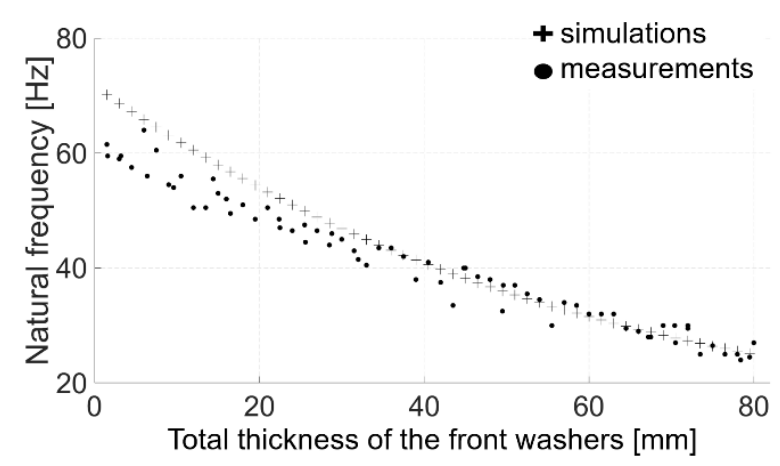

Figure 13. Metal discs considered as a single cylinder with an isotropic material (Young's modulus 11 [MPa])

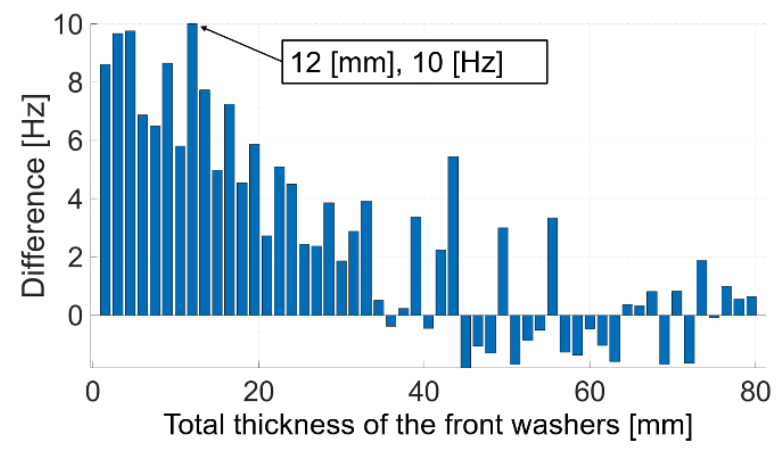

Figure 14. Differences between the simulations and the measurements

\subsection{Metal discs considered as a single cylinder consisting of an orthotropic material}

In this subsection, the metal discs are considered as a single cylinder consisting of an orthotropic material. This concept is inspired by the results of the last subsection.

The governing equation of the orthotropic material is

$$
\left[\begin{array}{l}
\varepsilon_{11} \\
\varepsilon_{22} \\
\varepsilon_{33} \\
\varepsilon_{12} \\
\varepsilon_{13} \\
\varepsilon_{23}
\end{array}\right]=\left[\begin{array}{cccccc}
\frac{1}{E_{1}} & \frac{-v_{12}}{E_{2}} & \frac{-v_{13}}{E_{3}} & 0 & 0 & 0 \\
\frac{-v_{12}}{E_{1}} & \frac{1}{E_{2}} & \frac{-v_{23}}{E_{3}} & 0 & 0 & 0 \\
\frac{-v_{13}}{E_{1}} & \frac{-v_{23}}{E_{2}} & \frac{1}{E_{3}} & 0 & 0 & 0 \\
0 & 0 & 0 & \frac{1}{G_{12}} & 0 & 0 \\
0 & 0 & 0 & 0 & \frac{1}{G_{13}} & 0 \\
0 & 0 & 0 & 0 & 0 & \frac{1}{G_{23}}
\end{array}\right]\left[\begin{array}{c}
\sigma_{11} \\
\sigma_{22} \\
\sigma_{33} \\
\sigma_{12} \\
\sigma_{13} \\
\sigma_{23}
\end{array}\right] .
$$

The $\varepsilon$ and the $\sigma$ are the strain and the stress. The elastic properties are completely defined by giving the Young's modulus
$E$, the shear modulus $G$ and the Poisson's ratio $v .9$ parameters should be defined to govern the material deformation. [Staab 2015]

The metal discs can be simulated more detailed using the orthotropic material than using the isotropic material. Thereby the limitation of the isotropic material can be eliminated. The parametrization bases on the 3 measurements (M1, M27 and M53) as shown in Fig. 3, the obtained parameters are listed in Tab. 1. Fig. 15 shows the orientations used for the material definition.

\begin{tabular}{|c|l|l}
\hline Parameter & Value & Unit \\
\hline$E_{1}$ & 5 & {$[\mathrm{MPa}]$} \\
\hline$E_{2}$ & 5 & {$[\mathrm{MPa}]$} \\
\hline$E_{3}$ & 5 & {$[\mathrm{MPa}]$} \\
\hline$G_{12}$ & 50 & {$[\mathrm{MPa}]$} \\
\hline$G_{13}$ & 10 & {$[\mathrm{MPa}]$} \\
\hline$G_{23}$ & 10 & {$[\mathrm{MPa}]$} \\
\hline$v_{12}$ & 0 & {$[-]$} \\
\hline$v_{13}$ & 0 & {$[-]$} \\
\hline$v_{23}$ & 0 & {$[-]$} \\
\hline
\end{tabular}

Table 1. Used parameters for the orthotropic material

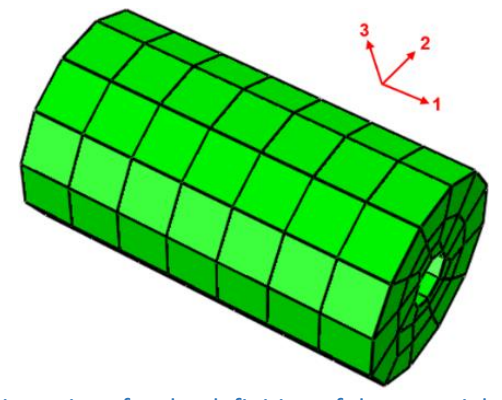

Figure 15. Orientations for the definition of the material

Same as in the last two subsections, the simulations are carried out for the 53 TMDDs. The first vibration mode of the model is shown in Fig. 9, it is the first bending mode of the screw. The natural frequency of this mode corresponds to the measured natural frequency in section 2. As shown in Fig. 15 and 16, the maximum difference between the simulations and the measurements $5.7[\mathrm{~Hz}]$ occurs with the total thickness of the front washers $12[\mathrm{~mm}]$, the average difference is $1.8[\mathrm{~Hz}]$. This model is parameterized with only 3 measurements and provides better simulation results than the other two models, and the average difference $1.8[\mathrm{~Hz}]$ lies in the tolerance range $3[\mathrm{~Hz}]$, which means the problems of the last two models have been solved.

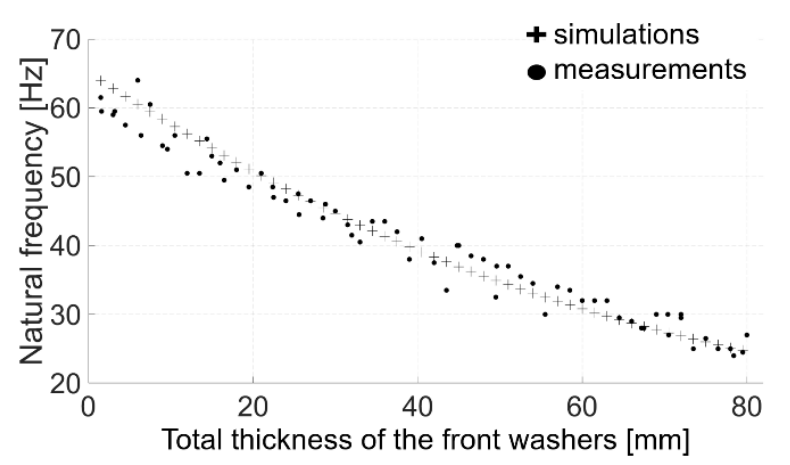

Figure 16. Metal discs considered as a single cylinder with anisotropic material 


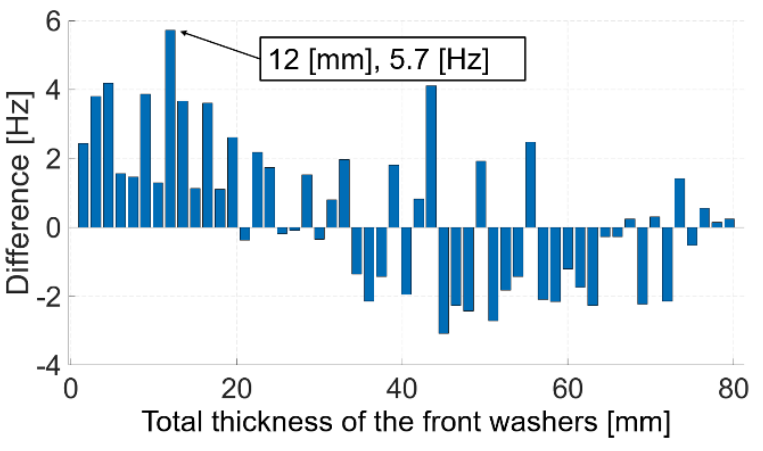

Figure 17. Differences between the simulations and the measurements

\subsection{The applicability of the third model}

The third simulation model proposed in the last subsection can predict the natural frequencies of the TMDDs with $600[\mathrm{~g}]$ vibration mass. The applicability of the model for the TMDDs with different vibration mass is proved in this subsection. Firstly, the natural frequencies of the three TMDDs shown in Fig. 18 are measured with the same measurement setup as demonstrated in Fig. 2. The simulation model is subsequently built as proposed in the last subsection. The material of the metal discs is parameterized based on the measurement M5.

The differences between the simulated and the measured natural frequencies (M4 2.8 [Hz], M5 $0.6[\mathrm{~Hz}], \mathrm{M} 61.0[\mathrm{~Hz}])$ are in the tolerance range $3[\mathrm{~Hz}]$, which means the model proposed in the last subsction is applicable for the TMDDs with different vibration mass.

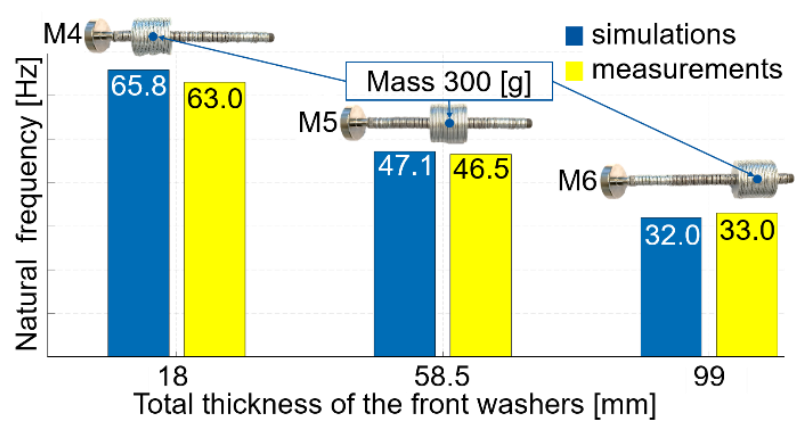

Figure 18. TMDDs with $300[\mathrm{~g}]$ vibration mass

\section{CONCLUSIONS}

Three finite element models of the TMDD are presented in this paper. The first model considers the metal discs as distributed mass along the screw. The simulated natural frequencies using this model are higher than the measured results, which lies in the incorrect modelling of the center of mass. The second model abstracts the metal discs as a single cylinder consisting of an isotropic material. This model considers the center of mass correctly and provides a better result than the first model. However this model increase the stiffness of the model artificially, therefore the simulated results are still higher than the measured results. This effect can be partially compensate by choosing one small Young's modulus for the single cylinder, however the Young's modulus can not be arbitrarily small due to the mapping problem between the simulations and the measurements. The third model considers the metal discs as a single cylinder consisting of an orthotropic material. The problems in the first and the second models have been solved in the third model. Therefore the third model can provide the best simulation results among these three models. The average difference between the simulated and the measured natural frequencies is $1.8[\mathrm{~Hz}]$, which lies in the tolerance range $3[\mathrm{~Hz}]$. Furthermore, the third model is applicable for the TMDDs with different vibration mass.

The natural frequencies of the TMDDs with the metal discs at the different positions can be predicted by the third finite element model, which can be parameterized with only few measurements and avoids the detailed simulation of the metal discs by abstracting them as a cylinder consisting of an orthotropic material. This model can accelerate the development process. In the future, multiple TMDDs will be applied during one portal milling process to validate their effectiveness.

\section{ACKNOWLEDGMENTS}

The authors would like to thank the German Research Foundation (DFG) for supporting this research under Grant No. BR2905/85-1: "Einsatz raeumlich verteilter passiver Daempfersysteme in einer Werkzeugmaschine".

\section{REFERENCES}

[Brecher 2020] Brecher, C., Zhao, G. and Fey, M., Design of a tuned mass disc damper with variable natural frequency for chatter suppression. Kaunas, Lithuania: Vibroengineering PROCEDIA, Vol. 34, pp. 39-42.

[Brecher 2017] Brecher, C. and Weck, M., Machine tools production systems 2. Germany: Springer, 2017. ISBN 978-3-662-46566-0

[Frahm 1911] Frahm, H., Frahm, H., 1911. Device for damping vibrations of bodies. U.S. Patent 989,958.

[Friend 2000] Friend, R.D. and Kinra, V.K., Particle impact damping. Journal of Sound and Vibration, 2000, Vol 233, No.1., pp.93-118., ISSN 0022-460X

[Hahn 1951] Hahn, R. S., Design of Lanchester damper for elimination of metal cutting chatter, Trans. ASME, 1951, Vol. 73, pp 331-331

[Li 2017] Li, S. and Tang, J., On vibration suppression and energy dissipation using tuned mass particle damper. ASME, 2017, Vol 139, No.1.

[Liu 2020] Liu, S., Lu, Z., Li, P., Zhang, W. and Taciroglu, E., Effectiveness of particle tuned mass damper devices for pile-supported multi-story frames under seismic excitations. Struct Control Health Monit, 2020, Vol 27, No.11, ISSN 1545-2263

[Lu 2020] Lu, Z., Masri, S.F. and Lu, X., Particle Damping Technology Based Structural Control. Singapore: Springer, 2020. ISBN 978-981-15-3499-7

[Meyer 2020] Meyer, N. and Seifried, R., Numerical and experimental investigations in the damping behavior of particle dampers attached to a vibrating structure. Computers \& Structures, 2020, Vol 238, ISSN 00457949

[Munoa 2016] Munoa, J., Beudaert, X., Dombovari, Z., Altintas, Y., Budak, E., Brecher, C. and Stepan, G., Chatter suppression techniques in metal cutting. CIRP Annals, 2016, Vol 65, No.2, pp.785-808., ISSN 00078506

[Staab 2015] Staab, G., Laminar composites. USA: ButterworthHeinemann, 2015. ISBN 978-0-12-802400-3

[Taylor 1906] Taylor, F. W., On the art of cutting metals. New York: American society of mechanical engineers, 1906. 


\section{CONTACTS:}

M.Sc. Guifeng Zhao

RWTH Aachen University

Laboratory for Machine Tools and Production Engineering

Steinbachstraße 19, Aachen, 52074, Germany

$+492418028235$

G.Zhao@wzl.rwth-aachen.de 